\title{
Lo que no sabíamos... \\ El movimiento sindical de Tetiz, Yucatán
}

\author{
Isela Rodríguez Alonzo
}

$\mathrm{E}$ n el año de 1990 se registró en Yucatán un importante episodio en la historia reciente de los movimientos sindicales y de resistencia indígena. Se trata de la lucha que llevaron al cabo los trabajadores avícolas de los pueblos de Tetiz y Hunucmá (dos municipios del estado de Yucatán colindantes al poniente con
Fernández" por el reconocimiento de su derecho a la libre sindicalización. Lo que distinguió este movimiento de otras luchas sindicales fue el involucramiento directo de todo el pueblo de Tetiz y gran parte del de Hunucmá con sus formas indígenas de resistencia.

Tetiz, bastión principal de los hechos, contaba en 1990 con 3444 habitantes, con un 52.6\% de población mayahablante mayor a los cinco años y un $19.5 \%$ de analfabetismo. El índice de escolaridad era muy bajo pues el $31 \%$ no contaba con instrucción alguna y sólo había concluido la primaria el $31 \%$. Este municipio estaba gobernado por un ayuntamiento emanado del Partido Acción Nacional, producto de un movimiento popular que en 1987 puso fin a décadas de dominio del Partido Revolucionario Institucional. Timoteo Canché Tinal, además de ser el alcalde y un destacado líder comunitario, había sido dirigente y fundador del independiente Sindicato de
Trabajadores de la Industria Avícola (siendo trabajador de otra empresa del ramo), mismo al que se afiliaron los trabajadores de "Avícola Fernández". Así se explica, al menos en parte, el papel clave protagonizado por este personaje y su posición comprometida con la voluntad de resistencia de su pueblo, a pesar de todas las presiones a las que fue sometido.

El dueño de la empresa se jactaba públicamente de su menosprecio a la demanda de un trato digno para los trabajadores mayas. Son precisamente las humillaciones, el maltrato y las vejaciones cotidianas, más que las condiciones laborales inhumanas, las causas que llevan a los trabajadores a organizarse. La respuesta empresarial es el despido de decenas de trabajadores, con lo que da inicio el conflicto que duraría cinco meses, durante los cuales hubo órdenes de aprehensión, cárcel, amenazas, intentos de soborno, una campaña de calumnias en los medios de comunicación y otras formas de represión. El conflicto rebasó la actuación del gobernador yucateco y requirió la intervención de la Secretaría de Gobernación.

Como asesores laborales participamos Julio Macossay Vallado, Mauricio Macossay Vallado y quien suscribe este testimonio. Julio estuvo preso 56 días, 50 de los cuales en huelga de hambre. Mauricio mantuvo la asesoría en

Desacatos, núm. 13, invierno 2003, pp. 170-174. 
Tetiz donde permaneció durante los últimos dos meses de la fase más crítica. Yo participé en las negociaciones con las autoridades laborales, la empresa y el gobierno del estado, a través de un periplo casi diario entre la cárcel, Tetiz y la ciudad de Mérida para mantener la coordinación entre los asesores y el pueblo.

Recién estrenado el año de 1990, llega una mañana a nuestro despacho de asesoría a sindicatos independientes Timoteo Canché a decirnos, con la calma que lo caracteriza y con el habla castellana con sintaxis maya de los indígenas yucatecos: "Me mandaron los trabajadores de las granjas de Fernández porque ya se cansaron y quieren su sindicato, ésta vez va en serio (cinco intentos anteriores resultaron frustrados), ya todos están seguros; se van a juntar pasado mañana en Tetiz pero a las 12 de la noche, quieren que sea clandestino para que la empresa no lo sepa antes que se metan los papeles, ya acordaron la asesoría y quieren que ustedes vayan en esa junta, yo los espero en el palacio".

Al llegar a Tetiz el día 10 de enero, en medio de una atmósfera de completo sigilo y cautela, con puntual precisión de movimientos nos llevan al solar de una casa retirada de la población. En plena oscuridad, al principio sólo distinguíamos a unos cuantos hombres alrededor del pozo, pero conforme se acostumbraba la vista empezamos a divisar, sorprendidos, las decenas de cabezas que se arremolinaban hasta no poder vislumbrar a los más alejados en la negrura de la noche. Era tan ostensible el silencio, tan sólo desafiado por el canto de los grillos, que uno pensaría que ahí no había un alma. Pero no, ahí estaban los 250 trabajadores mayas de las granjas de Tetiz y Hunucmá para participarnos su bien resguardado secreto y su determinación. Ahí escuchamos por primera vez el rosario de agravios y vejaciones que ya no estaban

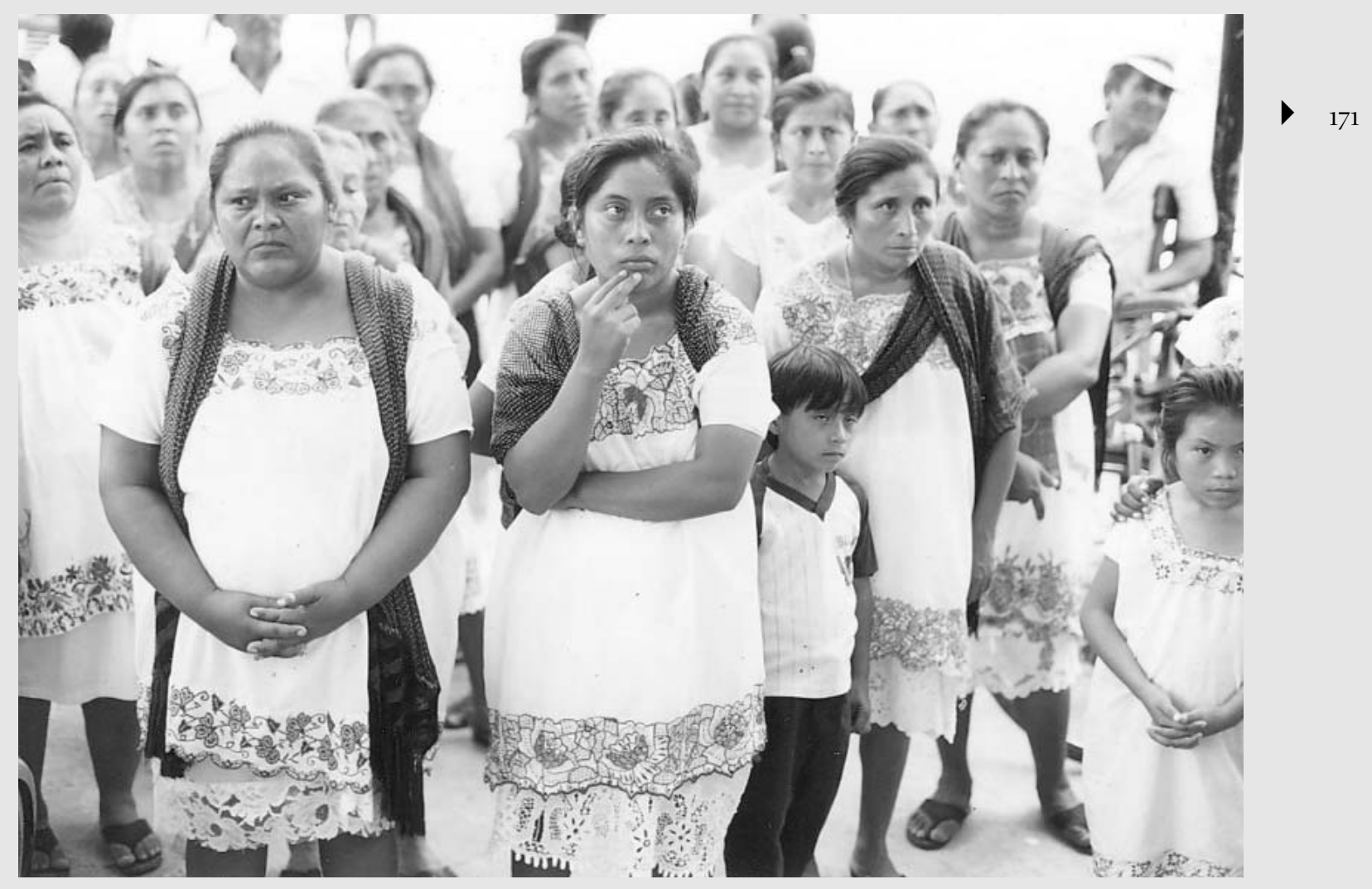

Movimiento de Tetiz, 1990 / Isela Rodríguez Alonzo. 
dispuestos a tolerar. Fue el primer indicio de que nos adentrábamos - más bien nos adentraban - en una dinámica de organización que más tenía que ver con ancestrales tradiciones indígenas que con prácticas obreras en sentido estricto. Así, en torno al brocal de un pozo, bajo las sombras de la noche, al amparo del monte y la húmeda frialdad del enero inaugural de la última década del siglo XX, da inicio este episodio de heroica resistencia.

Lo que no sabíamos entonces era que detrás del conjunto de trabajadores reunidos esa noche estaba todo el pueblo de Tetiz. En este testimonio quiero dejar constancia de lo que este pueblo maya nos enseñó - con todo y nuestros años de experiencia en luchas sindicales- en términos de conocimientos, intuición, ingenio, sabiduría, formas organizativas heredadas de sus antepasados $y$, de manera deslumbrante, del sentido profundo de la dignidad. Rescato tan sólo algunos de los múltiples episodios ocurridos y que dan cuenta de las formas autónomas y atávicas de organizar y ejercer la resistencia del pueblo maya, más allá de los acuerdos sindicales propiamente dichos tomados en las asambleas. Todos los tratrabajado en la Avícola Fernández y conocían en carne propia el maltrato y las afrentas a la dignidad; de aquí que la colectividad hiciera suya la resistencia.

Al día siguiente del estallido de la huelga (por la reinstalación de los trabajadores despedidos ante la solicitud del sindicato independiente) llega un centenar de policías antimotines y esquiroles cetemistas a tomar una de las granjas a la entrada de Tetiz. Se echan a vuelo las campanas de la iglesia y de inmediato salen decenas de hombres y mujeres. El primero en llegar es el alcalde, quien reclama la violación a la autonomía municipal, pero sin escucharlo, los policías se precipitan a retirar la bandera rojinegra. Timoteo se interpone para impedirlo y queda acorralado entre la reja y las armas de la policía encañonándolo. El comandante ordena cortar cartucho y Timoteo sin pestañear se voltea hacia un reportero gráfico y le dice: "Ramón, toma la foto cuando disparen.” En ese momento llegan los teticeños que no sólo hacen huir a los antimotines, sino que detienen a varios esquiroles a quienes las mujeres dejan en paños menores y los escoltan hasta el calabozo, sin dejar de amenazarlos durante todo el tra-

yecto: "La próxima vez que vengan a violentar, les untamos popox (hierba urticante) en sus huevos, iiya lo oyeron?!" Varias mujeres toman ese día el primero de sus tantos acuerdos: "Aquí nos vamos a quedar a vivir con los niños a cuidar la huelga" (a la intemperie junto a la granja). Así lo hicieron y en una de nuestras visitas llegamos a este campamento pasada la media noche para hacer una asamblea urgente, la frialdad del monte calaba hasta los huesos y en medio de la atmósfera de expectación y avidez de información en el rostro de todos, sobresalía una imagen conmovedora: dispersos encima de las piedras gran cantidad de niños de todas las edades durmiendo, abrigados apenas con toallas raídas. Hombres, mujeres y niños permanecieron ahí los doce días que duró este primer momento del movimiento de resistencia.

En las negociaciones interviene el Centro Empresarial de Mérida y su presidente nos "pide" a los asesores hablar a solas con los trabajadores; nos alejamos sin intervenir pero escuchando el diálogo completo. El empresario les pronuncia un largo y florido discurso - con marcado tinte paternalista - acerca del Estado de derecho, del respeto a la legalidad y de condena a la anarquía que a todos perjudica en referencia a la situación de huelga. Termina exhortándolos a retomar el camino de la ley. Demetrio Koyoc Cuytún, un destacado dirigente, le responde al empresario en tono sereno y pausado:

Con el respeto que usted merece, señor licenciado, ¿̨ónde está la ley estos diez años que nos tratan como animales? ¿Dónde está la ley cuando le pedimos a don Jorge (Fernández) que respete y nomás nos regaña, nos insulta y nos dice gritado: «indios de "miarda" — perdonando la palabrasi no les gusta se pueden largar, hay muchos muertos de hambre para trabajar»? ¿ Dónde está la ley diez años que estamos trabajando en el lodo con gallinaza hasta los tobillos? Además, señor, nuestro salario no es como dice la ley, la chamba son diez horas diario y no hay (pago de horas) extras. Otra cosa, la carga de trabajo por día (cantidad de cajas de huevo) no podemos solos, tenemos que pagar de nuestra bolsa a los niños que nos ayudan, si no lo cumplimos como dice el patrón, a nos saca, y no es justo porque a los de Campi (otra empresa avícola) sí lo respetan la cuota que dice la ley. Siempre con el debido respeto, señor, todas las veces que queremos la ley, el patrón nos insulta, nos saca y si no firmamos lo que quiere (sin liquidación) nos acusa de robo en el Ministerio. La problema no nosotros la 
creamos, licenciado, por eso estamos haciendo esto [la huelga], para que se arregle esta problema porque ya nos fastidiamos. Queremos que la ley que usted dice, hoy sí nos haga caso.

Fue muy notorio el mutismo que invadió a los representantes empresariales y el presidente del CEMER sólo alcanzó a esbozar un: "Gracias, muchas gracias por su tiempo y ojalá que el problema se resuelva para bien de todos."

Culmina la huelga con la victoria al firmar la empresa el reconocimiento del sindicato independiente, pero no se sabía entonces que se trataba de un ardid del empresario para ganar tiempo y dar el golpe definitivo. En la madrugada del 4 de abril alrededor de 200 esquiroles y pandilleros, armados de cadenas, chacos y pistolas irrumpen en las granjas para tomarlas. Pero una vez más el pueblo de Tetiz lo impide, obliga a huir a los pandilleros hasta las afueras del pueblo y toma prisioneros a los capataces de la empresa; las mujeres los desnudan y cumplen su amenaza de untarles popox en los testículos. Timoteo los detiene en el calabozo municipal para salvarlos del linchamiento y los consigna a la capital del estado donde las autoridades los liberan enseguida. Entretanto, el asesor legal Julio Macossay es encarcelado en Mérida y se giran 25 órdenes de aprehensión contra los dirigentes. Estos hechos marcan el inicio de la última y más dura etapa, del 5 de abril al 2 de junio. La campaña de amenazas y agresiones se agudiza y a mediados de mayo encarcelan a nueve trabajadores. La empresa utiliza toda su influencia para tratar de aplastar la resistencia. Las mujeres conminan a los trabajadores a no rendirse: "Tenemos escuchado en el radio lo que dijo Fernández, que los de Tetiz son puros huiros que sólo saben hacer hijos, ¿quién es culero para hincarse a Fernández?" Los hombres responden: "Si las mujeres no se rajan, nosotros tampoco."

Cuando el otro asesor, Mauricio Macossay, propone organizar la defensa para evitar las detenciones y la amenaza de nuevas agresiones, los teticeños le contestan: "Eso ya estuvo, ya está listo las postas, cada doce horas tienen su relevo, todo el perímetro del pueblo lo tenemos cubierto, tenemos vigilado quién entra y quién sale." A pregunta expresa refieren que son estrategias que aprendieron de sus antepasados. También por acuerdo comunitario le ponen custodios al asesor las 24 horas del día. En una ocasión, ante el riesgo de una posible detención del asesor, un importante dirigente de la comunidad le hace entrega de un rifle calibre 22 al tiempo que le dice: "Si es necesario dispara, yo me hago responsable."

$\mathrm{Al}$ amanecer del 26 de abril arriban a Tetiz más de 400 antimotines armados con rifles de alto poder y chalecos antibalas y acordonan la plaza y el atrio de la iglesia para impedir el acceso a la campana. Dos teticeñas rompen el cordón policiaco a codazos y empujones y se dirigen a tocarla. Al tratar de impedirlo, los policías las encañonan y les gritan: “¡No avancen o disparamos!” Sin más, las mujeres responden: “¡Mátanos, pero la campana la vamos a tocar!" Y diciendo y haciendo se cuelgan de la cuerda llamando al pueblo. Entretanto, las pocas venteras de verduras que se hallan cercadas en la plaza no dejan de increpar al jefe de la policía, quien enardecido les grita: “ ¡Todas las de Tetiz están fichadas!”, y una de ellas le contesta a voz en cuello: “¡Y tu puta madre, ¿no está fichada?!" Simultáneamente llegan otras mujeres que rompen el cerco a empujones y cuando los policías les apuntan, levantan los brazos y los desafían: “¡Disparen cobardes, no tenemos armas, véanlo!" Cuando el jefe de la policía ve que el pueblo empieza a salir de sus casas, visiblemente alterado les grita a sus subordinados: “iVámonos, vámonos!” En la huida son apedreadas las patrullas y las camionetas antimotines. Varios policías resultan descalabrados por los niños que agazapados detrás del muro de la iglesia no cesan de disparar proyectiles de piedras con sus tirahules. Es indudable que la policía no tenía órdenes de disparar, pero no lo sabían en esos momentos los hombres y mujeres que resistieron con coraje. Ante cada nueva agresión, y sin lograr la aceptación del sindicato ni la liberación de los presos (el asesor encarcelado se acercaba a los 50 días en huelga de hambre), las mujeres exclaman con rabia: “ ¡Vamos a quemar las granjas, para que aprenda Fernández que en Tetiz sí tenemos huevos!"

Finalmente, el gobierno de Salinas de Gortari, a través de la Secretaría de Gobernación, plantea el ultimatum: los trabajadores se desisten del sindicato o envían al ejército a tomar las granjas. En una asamblea de todo el pueblo, los trabajadores acuerdan por unanimidad renunciar todos a la empresa y exigir la liberación de los presos: "Preferimos morirnos de hambre antes que bajar nuestro 
pantalón a Fernández." Las mujeres toman su acuerdo: "Si nos engañan otra vez y no sacan a los presos, nos sentamos con nuestros hijos en las granjas a esperar a los soldados." Por dignidad decidieron iniciar la lucha y por dignidad decidieron cómo concluirla. Una vez más nos sorprendían con lo inédito de sus acuerdos: ¿̨cuándo habíamos visto que un conflicto obrero-patronal termine con la renuncia voluntaria de todos los trabajadores? Después de su salida, los trabajadores nos confiaron su último, callado y furtivo acto de resistencia: "Le dejamos su regalo a Fernández porque antes que le entregamos las granjas a los encargados, desnivelamos un poquito los caños de agua de las aves; además, ya se fastidió Fernández porque las aves se van a picotear cuando entre puro nuevo (trabajador) que no conocen."

Pero todavía nos esperaban varias sorpresas. Al llegar a Tetiz con los presos liberados pero con la sensación de una derrota sindical en toda la línea, los cientos de hombres, mujeres, niños y ancianos que nos esperaban en el cabo del pueblo, sencilla y llanamente andaban de fiesta, rebosando de alegría, celebrando la victoria con los puños en alto y lágrimas de júbilo en los ojos. Retumbaba

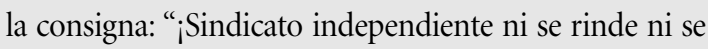
vende!” ¿Cuál victoria?, nos preguntábamos los asesores. Más aún, pensando que la liberación de los presos se logró gracias a la heroica resistencia, nos dejaron bien claro que estábamos equivocados: "No, no fue por eso doña Isela, salieron porque así lo quiso la Virgen, porque diario le rezamos y le ponemos veladora."

Dos días después se celebró una misa de acción de gracias en la iglesia de Tetiz, concelebrada por los dos sacerdotes que apoyaron decididamente la resistencia. ¿Victoria? ¿Acción de gracias? De manera insospechada el sermón del párroco de Tetiz nos dio las respuestas: "Esta es una misa del triunfo de la libertad, la justicia y la dignidad de un pueblo, un pueblo de hombres y mujeres que no se arrodillaron ante nada ni ante nadie." Después de la misa, para seguir festejando la victoria de la dignidad, arrancó el baile de puras cumbias hasta recibir el sol del nuevo día, de pie, bailando y con la frente en alto.

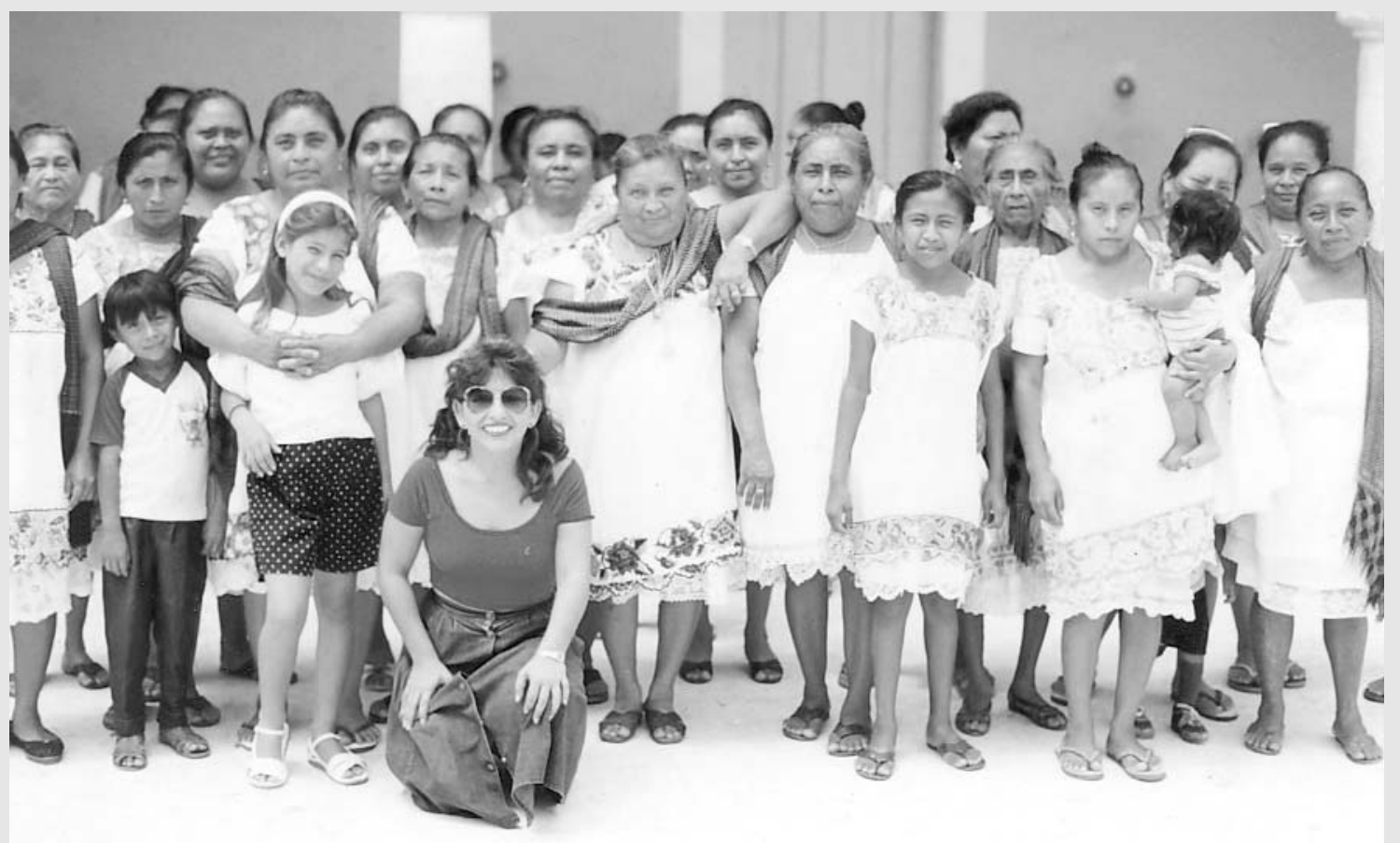

Mujeres del movimiento de Tetiz junto a Isela Rodríguez Alonzo, 1990 / Isela Rodríguez Alonzo. 